\title{
Triplet excitations in the frustrated spin ladder $\mathrm{Li}_{2} \mathrm{Cu}_{2} \mathrm{O}\left(\mathrm{SO}_{4}\right)_{2}$
}

\author{
O. Vaccarelli, ${ }^{1}$ A. Honecker ${ }^{2}$ P. Giura,${ }^{1}$ K. Béneut,${ }^{1}$ B. Fåk,${ }^{3}$ G. Rousse,${ }^{4}$ and G. Radtke ${ }^{1, *}$ \\ ${ }^{1}$ Sorbonne Université, UMR CNRS 7590, Muséum National d'Histoire Naturelle, \\ IRD UMR 206, Institut de Minéralogie, de Physique des Matériaux, \\ et de Cosmochimie (IMPMC), 4 place Jussieu, F-75005 Paris, France \\ ${ }^{2}$ Laboratoire de Physique Théorique et Modélisation, CNRS UMR 8089, \\ Université de Cergy-Pontoise, F-95302 Cergy-Pontoise Cedex, France \\ ${ }^{3}$ Institut Laue-Langevin, CS 20156, F-38042 Grenoble Cedex 9, France \\ ${ }^{4}$ Collège de France, Chimie du Solide et de l'Energie, UMR 8260, \\ 11 place Marcelin Berthelot, F-75231 Paris Cedex 05, France
}

\begin{abstract}
Magnetic excitations of the recently discovered frustrated spin-1/2 two-leg ladder system $\mathrm{Li}_{2} \mathrm{Cu}_{2} \mathrm{O}\left(\mathrm{SO}_{4}\right)_{2}$ are investigated using inelastic neutron scattering, magnetic susceptibility and infrared absorption measurements. Despite the presence of a magnetic dimerization concomitant with the tetragonal-to-triclinic structural distortion occurring below $125 \mathrm{~K}$, neutron scattering experiments reveal the presence of dispersive triplet excitations above a spin gap of $\Delta=10.6 \mathrm{meV}$ at $1.5 \mathrm{~K}$, a value consistent with the estimates extracted from magnetic susceptibility. The likely detection of these spin excitations in infrared spectroscopy is explained by invoking a dynamic Dzyaloshinskii-Moriya mechanism in which light is coupled to the dimer singlet-to-triplet transition through an optical phonon. These results are qualitatively explained by exact diagonalization and higher-order perturbation calculations carried out on the basis of the dimerized spin Hamiltonian derived from first-principles.
\end{abstract}

\section{INTRODUCTION}

Frustrated spin-1/2 two-leg ladders form a class of models which has been the subject of intense theoretical interest during the last decades as it fulfills all the requirements favoring the emergence of exotic phenomena $^{1-7}$. True material realizations of these models, however, are very scarce in the literature as the stringent conditions defining the geometry of frustrated ladders are rarely met in natural or synthetic compounds.

A noticeable exception is $\mathrm{BiCu}_{2} \mathrm{PO}_{6}$, a system built from coupled spin-1/2 two-leg ladders where frustration arises from competing antiferromagnetic nearest and next-nearest neighbor interactions along the legs ${ }^{8}$. The rich physics emerging from this particular geometry has triggered many experimental investigations of the magnetic properties of this compound over the past decade. These studies reveal a spin-gap behavior ${ }^{8,9}$, frustrationinduced incommensurate dispersion of triplet quasiparticle excitations ${ }^{10,11}$, triplet dispersion renormalization resulting from a repulsion with the multi-quasiparticle continuum, ${ }^{12}$ or a cascade of field-induced phase transitions ${ }^{13}$ including, presumably, the formation and collapse of a quantum soliton lattice ${ }^{14,15}$.

A new material realization of a frustrated spin- $1 / 2$ twoleg ladder system, $\mathrm{Li}_{2} \mathrm{Cu}_{2} \mathrm{O}\left(\mathrm{SO}_{4}\right)_{2}$, has recently been discovered $^{16-18}$. At high temperature, this compound crystallizes in a tetragonal structure where $\left[\mathrm{Cu}_{2} \mathrm{O}\left(\mathrm{SO}_{4}\right)_{2}\right]^{2-}$ chains running along the $c$ axis are well separated by $\mathrm{Li}^{+}$ions, thus forming quasi-1D structural units (see Fig. 1(a) and (b)). A first-principle investigation of the electronic structure of this compound ${ }^{18}$ clearly identified these structural units as magnetically equivalent to frustrated two-leg ladders where, as in $\mathrm{BiCu}_{2} \mathrm{PO}_{6}$, frustration arises from competing interactions along the legs.
The geometry of this system, however, differs substantially from that of $\mathrm{BiCu}_{2} \mathrm{PO}_{6}$ because (i) inter-ladder interactions are very weak, (ii) the rung coupling $\left(J_{\perp}\right.$ in Fig. 1(c)) is presumably ferromagnetic and (iii) additional antiferromagnetic diagonal interactions $\left(J_{\times}\right.$in Fig. 1(c)) occur.

The phenomenology of this compound is also markedly different as a structural transition to a distorted triclinic phase occurs near $125 \mathrm{~K}^{17}$ (see Fig. 1(d)). Although lattice parameters are barely affected by this transition, large variations of the interplatelet $\mathrm{Cu}-\mathrm{O}-\mathrm{Cu}$ superexchange angles drastically impact the amplitudes of leg and diagonal interactions $J$ and $J_{\times}$. These couplings, equivalent by symmetry in the high-temperature phase, are largely split by the triclinic distortion, leading to the emergence of a dominant antiferromagnetic interaction $J^{d}$. The resulting structure, while maintaining the global geometry of a ladder, displays a strong magnetic dimerization, where dimers are staggered on the legs of the ladder, as shown in Fig. 1(e). This scenario, describing the evolution of a complex geometry with the temperature, is however essentially derived from first-principle calculations and a definite assessment of the model requires further experimental investigations.

In this work, we report the first detailed investigation of the low-temperature magnetic excitations of $\mathrm{Li}_{2} \mathrm{Cu}_{2} \mathrm{O}\left(\mathrm{SO}_{4}\right)_{2}$ combining inelastic neutron scattering (INS), magnetic susceptibility and infrared (IR) spectroscopy measurements carried out on powder samples. Dispersive triplet excitations are observed in INS above a spin gap $\Delta=10.6 \mathrm{meV}$ at $1.5 \mathrm{~K}$, a value consistent with the estimates extracted from magnetic susceptibility. Moreover, an absorption band visible only in the dimerized phase below $70 \mathrm{~K}$ is observed in IR spectroscopy and attributed to a triplet excitation arising at 

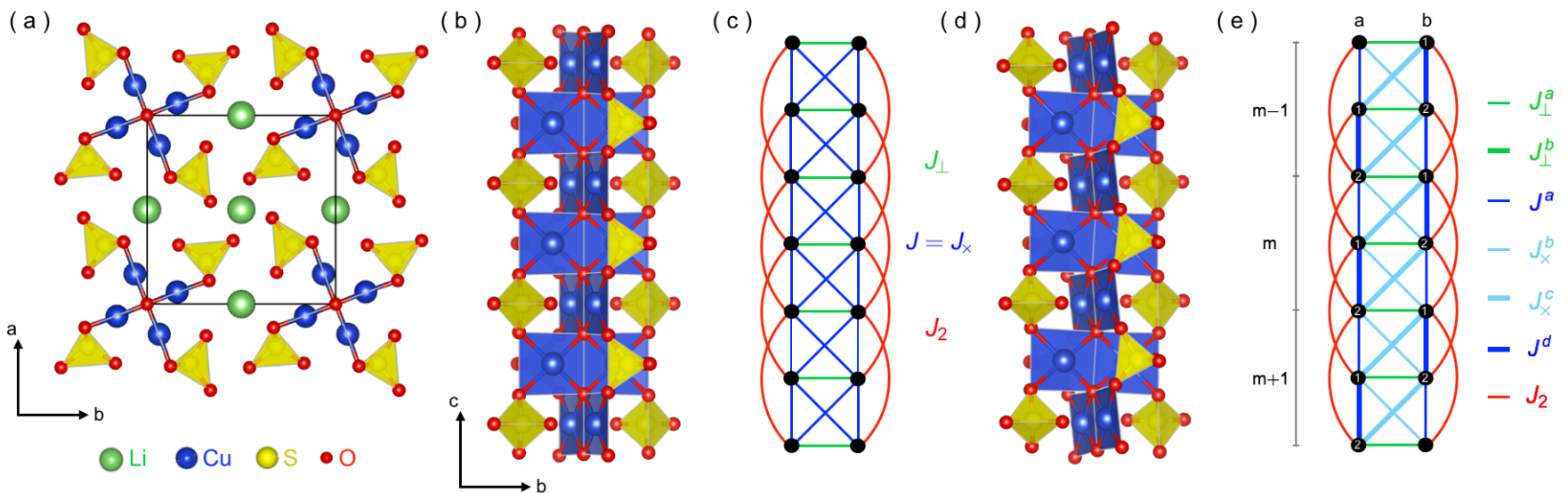

FIG. 1. (a) Structure of tetragonal $\mathrm{Li}_{2} \mathrm{Cu}_{2} \mathrm{O}\left(\mathrm{SO}_{4}\right)_{2}$ viewed along the [001] direction. (b) Structure of the $\left[\mathrm{Cu}_{2} \mathrm{O}\left(\mathrm{SO}_{4}\right)_{2}\right]^{2-}$ chains running along the $c$ axis in the high- temperature $(T>125 \mathrm{~K})$ phase. (c) Schematic representation of the corresponding spin system. Intra $\left[\mathrm{Cu}_{2} \mathrm{O}_{6}\right]^{8-}$ platelet rung coupling $J_{\perp}$ is represented in green, leg and diagonal couplings $J=J_{\times}$are

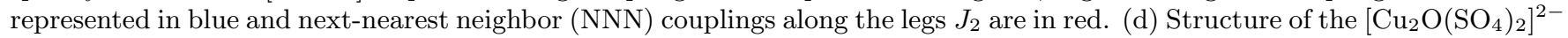
chains in the distorted low-temperature $(T<125 \mathrm{~K})$ phase. (e) Schematic representation of the corresponding staggered-dimer structure: $J^{a}$ and $J^{d}$ in blue represent the alternating couplings along the legs, $J_{\times}^{b}$ and $J_{\times}^{c}$ in light blue the diagonal inter-chain couplings, $J_{\perp}^{a}$ and $J_{\perp}^{b}$ in green the couplings between the chains along the rungs and the NNN coupling along the legs $J_{2}$ in red.

$14.3 \mathrm{meV}$. The dynamic Dzyaloshinskii-Moriya mechanism is invoked in this case to explain the absorption of light by this low-dimensional spin system. All these observations are qualitatively explained by higher-order perturbation and exact diagonalization calculations of triplet quasi-particle excitations carried out on the basis of the dimerized geometry derived from previous firstprinciple calculations ${ }^{18}$.

\section{EXPERIMENTS}

\section{A. Time-of-flight neutron spectroscopy}

Inelastic neutron scattering experiments were performed at the Institut Laue-Langevin in Grenoble on the thermal time-of-flight spectrometer IN4 using incoming energies of 16.6, 32.0, and $66.6 \mathrm{meV}$. Most measurements were done with an incoming energy of $32.0 \mathrm{meV}$ from the (004) reflection of a pyrolytic graphite monochromator. A Fermi chopper was used with a $2^{\circ}$ straight slit package spinning at $15000 \mathrm{rpm}$. The energy resolution (full width at half maximum) at elastic energy transfer was $2.1 \mathrm{meV}$. A total of $6.8 \mathrm{~g}$ of powder sample of $\mathrm{Li}_{2} \mathrm{Cu}_{2} \mathrm{O}\left(\mathrm{SO}_{4}\right)_{2}$ was synthesized according to the procedure described in Ref. 16, put in a flat aluminum plate holder of dimensions of $40 \times 28 \times 2 \mathrm{~mm}^{3}$, and mounted in a standard helium cryostat. Measurements were carried out at temperatures $T=1.5,40,60,80$ and $100 \mathrm{~K}$ for a typical acquisition time of $12 \mathrm{~h}$ per temperature. The scattering from the empty Al-holder was measured and subtracted from the data, which was subsequently corrected for self-shielding and absorption using standard data reduction routines. Data treatments were carried out using the LAMP software ${ }^{19}$.

Figure 2(a)-(e) shows the maps of the dynamic susceptibility $\chi^{\prime \prime}(|Q|, E)$, obtained by normalizing the background-subtracted neutron scattering intensity $S(|Q|, E)$ by the thermal occupancy factor $1-e^{-E / k_{\mathrm{B}} T}$ at $1.5,40,60,80$, and $100 \mathrm{~K}$, respectively. The temperature dependence of the dynamic susceptibility clearly reveals the presence of two dominant contributions arising from phonon and magnetic excitations. Whereas the scattering cross-section of the former scales as $|Q|^{2}$, that arising from magnetism scales with the square of the form factor associated with the magnetic ions, and falls off with increasing $|Q|^{20,21}$. The high-temperature dynamic susceptibility is therefore largely dominated by phonon scattering (see Fig. 2(d)-(e)) whereas the weight of the magnetic contribution progressively increases with decreasing temperatures, as it can be observed in the low- $|Q|$ region of Fig. 2(a)-(c). Assuming, in first approximation, that the intensity is entirely associated with phonons scattering for the highest temperature measurement $(T=100 \mathrm{~K}$ in our case), the magnetic contribution to the dynamic susceptibility at low-temperature can simply be isolated by plotting the difference ${ }^{22}$

$$
\Delta \chi^{\prime \prime}(|Q|, E)=\left.\chi^{\prime \prime}(|Q|, E)\right|_{1.5 \mathrm{~K}}-\left.\chi^{\prime \prime}(|Q|, E)\right|_{100 \mathrm{~K}}
$$

The corresponding difference map is shown in Fig. 2(f) and clearly reveals the presence of dispersive magnetic excitations of bandwidth $\approx 5 \mathrm{meV}$ above a large spin gap $\Delta=10.6 \mathrm{meV}$, measured as the inflection point of the first peak (A). Constant- $Q$ cuts extracted for a $|Q|$ value of $2 \pm 0.1 \AA^{-1}$ for each temperature measured experimentally are shown in Fig. 3. The broad band of magnetic excitations, extending over $5 \mathrm{meV}$ above the 


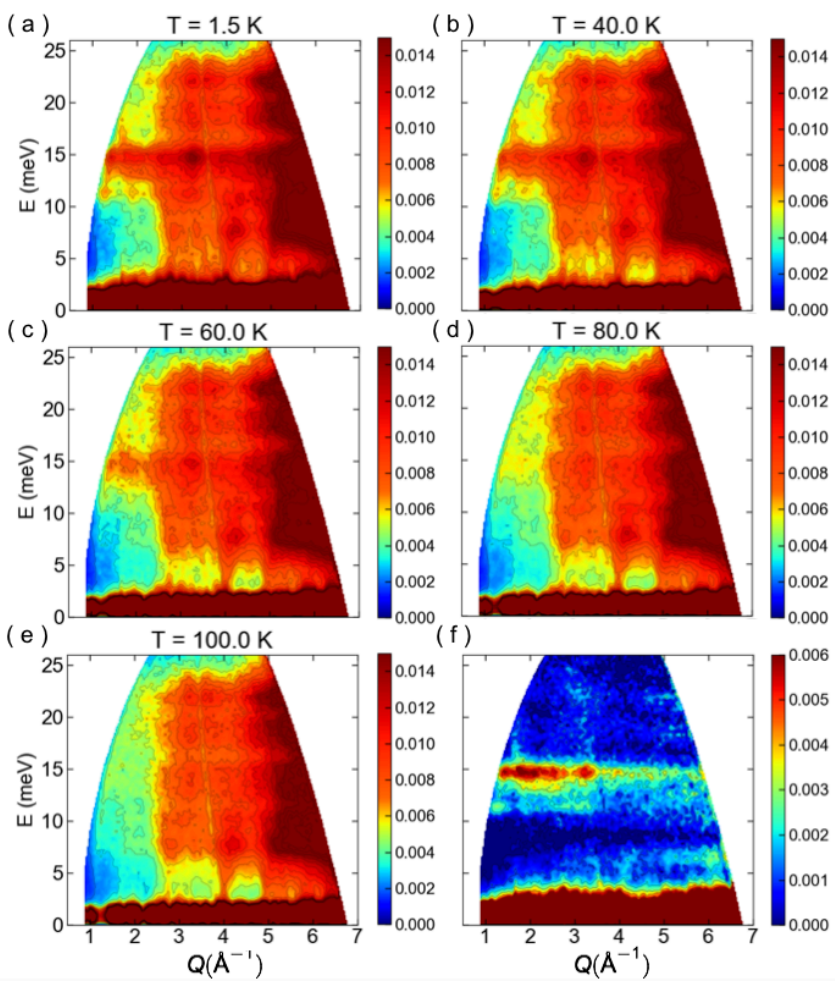

FIG. 2. Experimental dynamic susceptibility $\chi^{\prime \prime}(|Q|, E)$ plots for $\mathrm{Li}_{2} \mathrm{Cu}_{2} \mathrm{O}\left(\mathrm{SO}_{4}\right)_{2}$ measured at different temperatures: (a) $T=1.5 \mathrm{~K}$, (b) $T=40 \mathrm{~K}$, (c) $T=60 \mathrm{~K}$, (d) $T=80 \mathrm{~K}$, and (e) $T=100 \mathrm{~K}$. Magnetic contributions are isolated in (f) by displaying the difference $\Delta \chi^{\prime \prime}(|Q|, E)$ from Eq. (1).

spin gap, includes the first low-intensity peak (A) centered at $11.2 \mathrm{meV}$ followed by the most intense structure (C) at $14.7 \mathrm{meV}$. A low-energy shoulder (B) of this dominant structure is also present at about $13.5 \mathrm{meV}$, particularly visible on the data obtained at $1.5 \mathrm{~K}$. Inelastic neutron scattering experiments therefore clearly reveal the presence of dispersive magnetic excitations, mostly localized at low- $|Q|<3.5 \AA^{-1}$ and vanishing at temperatures above $\sim 80 \mathrm{~K}$, consistent with spin triplet excitations out of the reported singlet ground state of this compound $^{17,18}$.

\section{B. Static magnetic susceptibility}

These results can be further confirmed by analyzing the low-temperature behavior of the experimental static magnetic susceptibility. Fig. 4 reproduces the experimental data corrected from paramagnetic impurities and temperature-independent contributions, already reported in Ref. 17. The susceptibility exhibits the typical behavior of a gapped low-dimensional antiferromagnet with a high-temperature paramagnetic regime reaching a broad maximum at about $125 \mathrm{~K}$ and an exponential decay at low-temperature. Note, however, that the

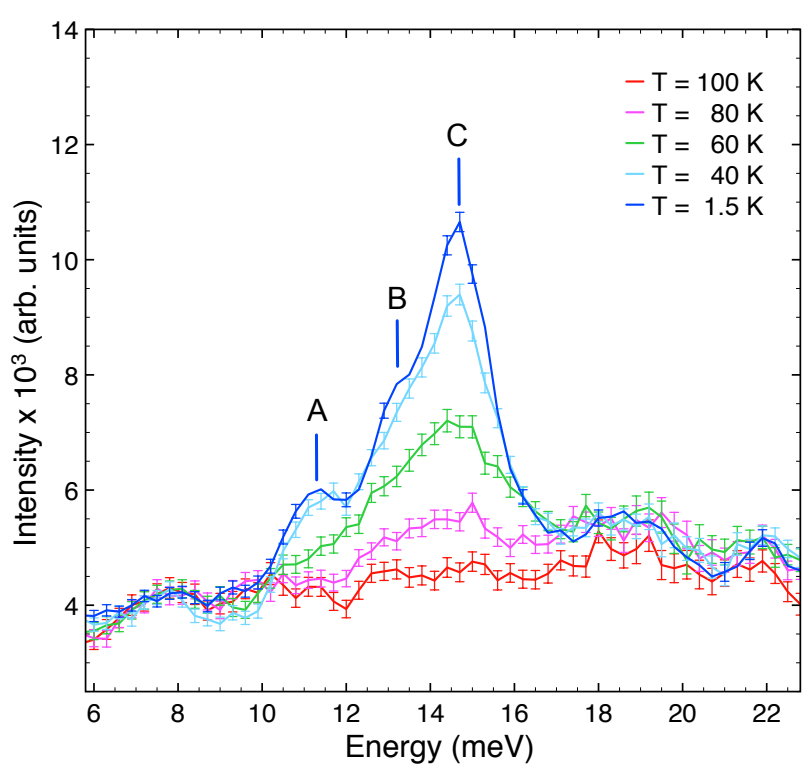

FIG. 3. Constant- $Q$ cuts of the dynamic susceptibility $\chi^{\prime \prime}(|Q|, E)$ extracted for a $|Q|$ value of $2 \pm 0.1 \AA^{-1}$ from the plots shown in Fig. 2(a)-(e).

temperature dependence of the magnetic susceptibility is largely affected by the structural transition occurring at the same temperature as the maximum $(125 \mathrm{~K})$ since it is accompanied by a substantial magnetic dimerization within the ladders. As reported in Ref. 18, although this transition extends over a large temperature range, the low-temperature triclinic phase is already mostly stabilized at about $80 \mathrm{~K}$, and the $2-80 \mathrm{~K}$ range can therefore be used to estimate the corresponding spin gap. A rough estimate can be obtained by fitting the experimental data using the general expression for thermally activated processes

$$
\chi(T) \propto e^{-\Delta / T}
$$

leading to a value of $\Delta \approx 9.1 \mathrm{meV}$. It should however be noted that (2) would be valid for non-interacting particles, but magnons are not free particles. A suitable expression that takes the hard-core repulsion between the magnons into account is given by ${ }^{23}$

$$
\chi_{\text {lad. }}(T) \propto T^{-1 / 2} e^{-\Delta / T}
$$

for a quadratic band minimum. Equation (3) has also been employed (see Fig. 4). The resulting estimate for the spin gap, $\Delta \approx 11.6 \mathrm{meV}$, is slightly larger than the value obtained with the previous expression. Despite these small variations, essentially related to the rather low accuracy of this approach and to the limited applicability of the simple ladder model to $\mathrm{Li}_{2} \mathrm{Cu}_{2} \mathrm{O}\left(\mathrm{SO}_{4}\right)_{2}$, these estimates are however fully consistent with the spin gap value obtained from inelastic neutron scattering. 


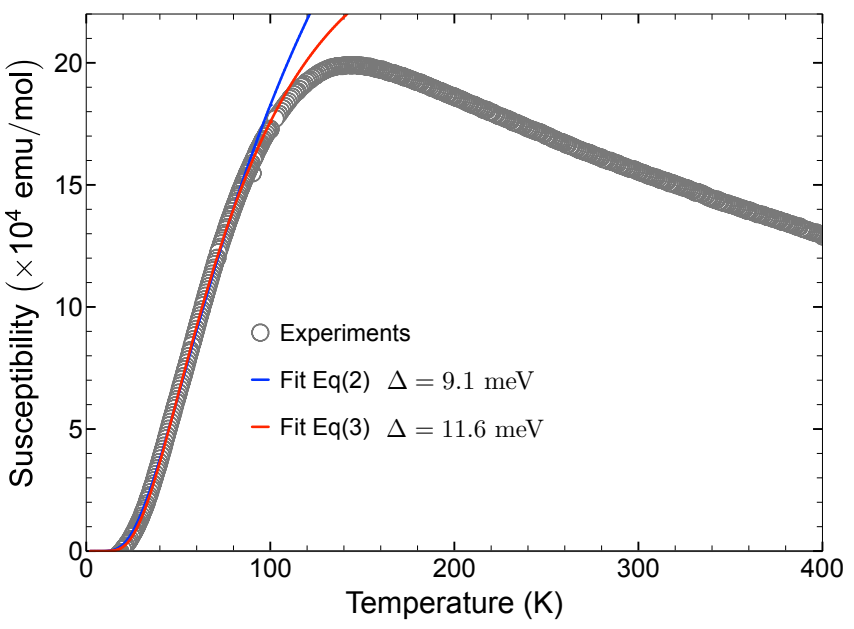

FIG. 4. Temperature dependence of the static magnetic susceptibility of $\mathrm{Li}_{2} \mathrm{Cu}_{2} \mathrm{O}\left(\mathrm{SO}_{4}\right)_{2}$ corrected from paramagnetic impurities and temperature-independent contributions. Grey circles correspond to the experimental points, the solid blue and red lines correspond, respectively, to the best fit obtained using the general expression (2) or a spin-1/2 two-leg ladder Heisenberg model (3). The corresponding values of the extracted spin gap are indicated.

\section{IR spectroscopy}

Infrared absorption spectroscopy was finally employed as a complementary technique to investigate the lowenergy excitations of $\mathrm{Li}_{2} \mathrm{Cu}_{2} \mathrm{O}\left(\mathrm{SO}_{4}\right)_{2}$. These experiments were carried out using a Fourier transform Bruker IFS66 v/s spectrometer at the IMPMC-Sorbonne University spectroscopy platform. The instrument was aligned in transmission geometry. Isotropic pellets of $\sim 13 \mathrm{~mm}$ diameter were prepared by mixing the original powder samples with transparent matrix materials. Pure $\mathrm{KBr}$ powder was used as a matrix for the pellets employed in the middle-infrared (MIR), whereas polyethylene (PE) was employed to prepare pellets for the far infrared (FIR) measurements. The former were obtained by mixing $2.5 \mathrm{mg}$ of sample and $80 \mathrm{mg}$ of pure PE; for the latter, $1.4 \mathrm{mg}$ of sample was mixed with $200 \mathrm{mg}$ of $\mathrm{KBr}$ powder, placed in an oven at $T=150^{\circ} \mathrm{C}$ in order to remove water contamination from the $\mathrm{KBr}$ powder, and then pressed to obtain high quality pellets. Transmission spectra were taken as a function of temperature from 10 to $300 \mathrm{~K}$ using a continuous Janis liquid helium cryostat working in vacuum. Each spectrum was acquired in the frequency region $60-640 \mathrm{~cm}^{-1}$ for the FIR measurements and $580-4400 \mathrm{~cm}^{-1}$ for the spectrum in the MIR, with a spectral resolution of about $2 \mathrm{~cm}^{-1}$. The FIR spectra were recorded with a DTGS-Pe detector and a multilayer mylar beamsplitter. The MIR data were obtained with a HgCdTe (MCT) detector and a Ge coated $\mathrm{KBr}$ beamsplitter. For both region we used a Globar ( $\mathrm{SiC})$ source.

The temperature dependence of the transmission IR

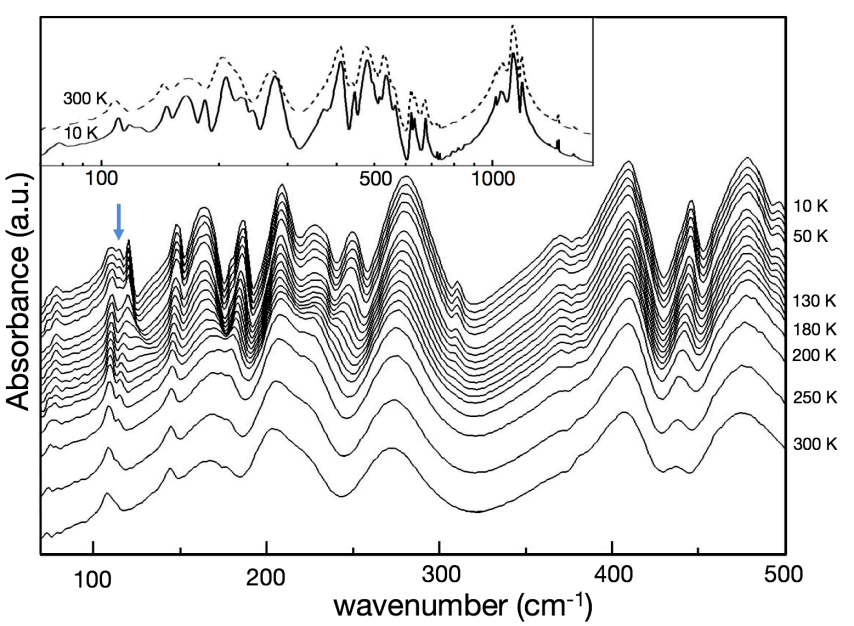

FIG. 5. Temperature dependence of the powder transmission infrared spectra of $\mathrm{Li}_{2} \mathrm{Cu}_{2} \mathrm{O}\left(\mathrm{SO}_{4}\right)_{2}$ in the FIR region. The blue arrow indicates the spectral feature associated to the magnetic excitation. Top inset: The lowest and highest temperature spectra are reported in logaritmic scale up to $1800 \mathrm{~cm}^{-1}$ to show the high energy modes associated with the internal $\left[\mathrm{SO}_{4}\right]^{2-}$ bond stretching.

powder spectrum of $\mathrm{Li}_{2} \mathrm{Cu}_{2} \mathrm{O}\left(\mathrm{SO}_{4}\right)_{2}$ measured between 10 and $300 \mathrm{~K}$ is shown in Fig. 5. Most of the absorption bands visible between 100 and $1300 \mathrm{~cm}^{-1}$ are associated with the electric dipole excitation of optical phonons. The group of high-energy modes located around $1100 \mathrm{~cm}^{-1}$ is exclusively associated with internal $\left[\mathrm{SO}_{4}\right]^{2-}$ bond stretching ${ }^{24}$. The $500-700 \mathrm{~cm}^{-1}$ range is dominated by $\left[\mathrm{SO}_{4}\right]^{2-}$ tetrahedra bending modes involving progressively the displacement of $\mathrm{Cu}$ and $\mathrm{O}$ atoms forming the chain backbone, as the frequency decreases.

Symmetry can be employed to determine the maximum number of infrared active optical phonons in both phases of this compound. In its high temperature phase, $\mathrm{Li}_{2} \mathrm{Cu}_{2} \mathrm{O}\left(\mathrm{SO}_{4}\right)_{2}$ belongs to the $P 4_{2} / m$ space group. A factor group analysis indicates that the vibrational degrees of freedom decompose as

$$
\Gamma_{\mathrm{HT}}=11 A_{g}+13 B_{g}+9 E_{g}+10 A_{u}+8 B_{u}+15 E_{u}
$$

on the irreducible representations of the $D_{4 h}$ point group. Subtracting the $A_{u}+E_{u}$ acoustic modes, we find 37 IR-active modes, decomposed in 23 potentially distinct $9 A_{u}+14 E_{u}$ bands. The triclinic distortion, occurring below $125 \mathrm{~K}$, further reduces the crystal symmetry to $P \overline{1}$. A similar analysis leads to the following decomposition on the only two irreducible representations of $C_{i}$

$$
\Gamma_{\mathrm{LT}}=42 A_{g}+48 A_{u}
$$

leading, after subtraction of the $3 A_{u}$ acoustic modes, to $45 A_{u}$ IR-active modes. As it can be observed in Fig. 5, the exact number of bands detected in these experiments is difficult to assess, due to the broad and asymmetric profile of certain peaks. The above group theoretical analysis therefore only provides an upper bound for 
the number of bands distinguishable in the experimental spectra. Qualitatively, however, the large increase of active modes due to the symmetry lowering triggered by the triclinic distortion is clearly visible on the experimental spectra when decreasing the temperature below the transition $(\sim 125 \mathrm{~K})$ and therefore consistent with the structural data ${ }^{17}$.

Besides this increase in the number of phonon lines, the main effects of decreasing the temperature consist in a slight hardening and narrowing of most of the bands, usually attributed with anharmonic effects and in particular, for the former, with the overall unit cell volume contraction $^{17}$. However, a few bands display a softening in the temperature range of the structural transition, characteristic of magneto-elastic effects associated in our case to the rise of the dimerization ${ }^{25}$. At low energy, however, a pronounced transfer of spectral weight toward low frequencies reveals the rise of a weak excitation at $115 \mathrm{~cm}^{-1}$ $(14.3 \mathrm{meV})$. This band is indicated by a blue arrow in Fig. 6(a).

In order to quantify these spectral changes, a leastsquare fit of the low-frequency range of the spectra based on a superposition of Lorentzians has been carried out. The temperature dependence of the energy of the different modes observed in the $105-152 \mathrm{~cm}^{-1}$ range resulting from this fit is displayed in Fig. 6(b). A weak hardening of the modes identified as polar phonons (grey filled diamonds) is visible with decreasing temperature except in the transition temperature range $(80-130 \mathrm{~K})$ where a sizable jump is observed. Concomitantly, the band shown in blue in Fig. 6(b) has an energy of $14.3 \mathrm{meV}$, that falls precisely in the broad band of magnetic excitations observed by INS, in a region characterized by a large spectral weight. Moreover, this excitation is only visible at temperatures well below the structural transition, i.e. in the magnetic dimerized phase. These observations therefore suggest that this excitation might involve, to a certain extent, the spin degrees of freedom of this system.

\section{THEORY}

In order to explain this set of experimental findings and explore the landscape of low-energy magnetic excitations in $\mathrm{Li}_{2} \mathrm{Cu}_{2} \mathrm{O}\left(\mathrm{SO}_{4}\right)_{2}$, both exact diagonalization and higher-order perturbation theory calculations have been carried out. The $Q$-dependence of the dynamic susceptibility $\chi^{\prime \prime}$ should be dominated by that of the $J^{d}$ dimers ${ }^{26}$, or more generally be related to the exchange constants via a first-moment sum rule ${ }^{27-30}$. However, any such analysis is complicated in $\mathrm{Li}_{2} \mathrm{Cu}_{2} \mathrm{O}\left(\mathrm{SO}_{4}\right)_{2}$ by the large phonon background. We have therefore decided to focus rather on the clear magnetic features $\mathrm{A}, \mathrm{B}$, and $\mathrm{C}$ visible in Fig. 3 by comparing to both exact diagonalization and higher-order perturbation theory calculations.

A realistic spin Hamiltonian (see Appendix A) capable of describing the magnetism of this compound in the

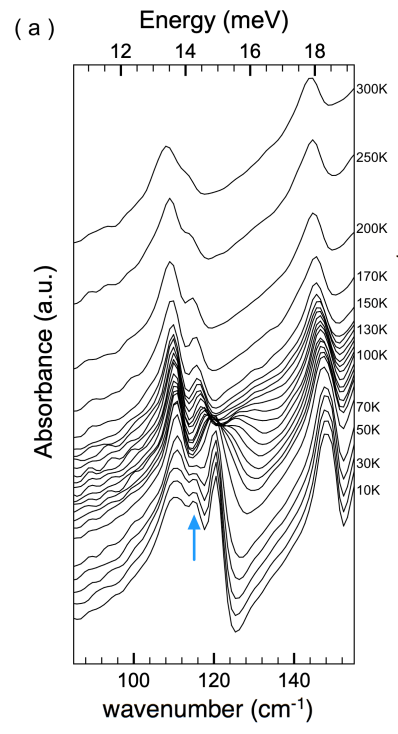

(b)

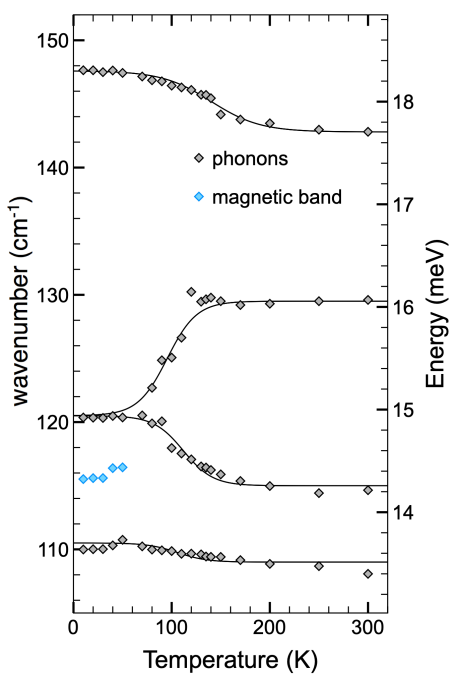

FIG. 6. (a) Enlarged view of the powder transmission infrared spectra in the $80-155 \mathrm{~cm}^{-1}$ range. (b) Temperaturedependence of the mode frequencies extracted from the Lorentzian fit. Experimental data are represented with filled diamond symbols, lines are only guides for the eye. The phonon bands are represented in black whereas the magnetic band is in blue.

triclinic phase has been derived previously ${ }^{18}$ from firstprinciple calculations and requires seven distinct couplings to account for the low symmetry of the crystal. The geometry of this model is depicted in Fig. 1(e) where $J^{d}=1$ and $J^{a}=20 / 330=2 / 33$ are antiferromagnetic and alternate along the legs of the ladder so as to form a staggered dimer structure, $J_{\perp}^{a} \approx J_{\perp}^{b}=-110 / 330=-1 / 3$ are the ferromagnetic couplings along the rungs of the ladder, $J_{\times}^{b}=78 / 330=13 / 55$ and $J_{\times}^{c}=133 / 330$ are antiferromagnetic diagonal couplings between the legs and, finally, $J_{2}=112 / 330=56 / 165$ is the antiferromagnetic NNN interaction along the legs. This model therefore neglects the supposedly very weak inter-ladder couplings ${ }^{18}$ as well as any other term beyond the bilinear, Heisenberg like, interactions.

Exact diagonalization (ED) calculations have been performed using finite lattices of $N=12,16,20,24,28$, and 32 sites with periodic boundary conditions along the legs, as the one-dimensional magnetic unit cell contains two dimers, i.e. four spins. For system sizes exceeding $N=20$ we have used the Lanczos algorithm ${ }^{31,32}$ in order to compute low-lying eigenvalues. Furthermore, perturbation expansion of the one-triplet dispersion relation up to the fifth order has been carried out around the limit of isolated dimers. In this approach, the unperturbed ground state corresponds to a product of singlets, $|s\rangle=(|\uparrow \downarrow\rangle-|\downarrow \uparrow\rangle) / \sqrt{2}$, on the leg dimers defined by the dominant antiferromagnetic coupling $J^{d}$. Lowenergy magnetic excitations of this system are obtained by promoting one dimer into a triplet state, $\left|t_{-1}\right\rangle=|\downarrow \downarrow\rangle$, $\left|t_{0}\right\rangle=(|\uparrow \downarrow\rangle+|\downarrow \uparrow\rangle) / \sqrt{2}$ or $\left|t_{1}\right\rangle=|\uparrow \uparrow\rangle$. Since the unit cell 
contains two dimers, we find two separate single-particle bands, as shown in Fig. 7 (see Appendix A for details).

Numerical results are summarized in Fig. 7. Fig. 7(a) and (b) show the convergence of the perturbation expansion by comparing, respectively, the one-triplet dispersion relations obtained at different expansion orders and the highest order perturbation theory with exact diagonalization results. As it clearly appears, a remarkable convergence towards the exact results is achieved for the higher-order expansions. These calculations indicate the presence of two dispersive and slightly overlapping triplet bands above a large spin gap. The lowest band displays a behavior characteristic of antiferromagnetically coupled dimers with a maximum at the zone center and a minimum close the Brillouin zone edge. The actual minimum arises at an incommensurate wave vector and results from the presence of frustrating couplings. In addition to the one-triplet excitation bands, the lower boundary of the two-triplet continuum is calculated as

$$
E_{2}(k)=\min _{\substack{q \in 1 \mathrm{BZ} \\ m, n=1,2}}\left[\omega_{m}(k-q)+w_{n}(q)\right]
$$

where $\omega_{1}(k)$ and $\omega_{2}(k)$ represent the two one-triplet bands, is also shown in Fig. 7(b). The large value of the spin gap compared to the modest triplet excitation bandwidth, pushes this continuum lower bound well above the maximum of the highest one-triplet band. The ED results are close to the fifth-order expansion, i.e., both of them can be considered accurate. The exception is the top of the upper band where proximity to the continuum leads to larger finite-size effects and slower convergence of the series (see Appendix B for details). Exact diagonalization, furthermore, reveals the presence of lower-lying singlets above and below the continuum, which do not interfere with the upper triplet band, as it can be seen in Fig. 7(b). Similar excitations have already been reported in ladder systems where they can be understood as bound states of two triplets ${ }^{8,33,34}$.

\section{DISCUSSION}

The theoretical results presented in the previous section provide solid ground for analyzing the experimental results obtained on $\mathrm{Li}_{2} \mathrm{Cu}_{2} \mathrm{O}\left(\mathrm{SO}_{4}\right)_{2}$. It should be noted, however, that the global energy scale obtained from firstprinciple calculations ${ }^{18}$ is not consistent with our experimental observations. Indeed, as already reported, a straightforward use of the magnetic couplings provided by density functional theory (DFT) calculations leads to a substantial overestimation of the experimental spin gap $^{18}$. Although the amplitudes of these couplings are often overestimated and strongly depend on the approximate treatment of exchange and correlation employed in the calculations ${ }^{35,36}$, their ratios are expected to be subject to smaller errors ${ }^{37}$. In this framework, the ratios between the seven couplings involved in the spin Hamiltonian were considered as fixed. The global energy scale was thus taken as the only variable parameter, adjusted so as to reproduce the experimental value of the spin gap. This led to an approximate $50 \%$ downscaling of the DFT coupling amplitudes. The resulting energy scale in millivolts is shown on the vertical axes of Fig 7(a) to (c).

Under these assumptions, powder INS can be qualitatively discussed in terms of one-triplet excitation densityof-states (DOS) shown in Fig. 7(c). In first approximation, the experimental peaks A, B and $\mathrm{C}$ shown in Fig. 3 can indeed be interpreted as arising from the DOS singularities, at the bottom of the lowest band for peak $\mathrm{A}$ and in the overlapping region of the two bands for peaks B (bottom of the upper band) and C (top of the lower band). Although this qualitative analysis provides a satisfying explanation regarding the origins of the lowenergy part of the INS data, it also predicts the presence of higher-lying features corresponding to the top of the highest one-triplet band, i.e. at $\sim 20 \mathrm{meV}$, which was not observed experimentally. This could simply be a matrix element effect. Alternatively, although our model locates the two-triplet continuum lower bound above the highest one-triplet branch over the entire first Brillouin zone (see Fig. 7(b)), they remain close in energy. Therefore, only minor modifications of the model employed in this work would be necessary to change this picture and, in particular, restore a significant overlap between the highest triplet quasi-particle mode and the two-particle continuum. This overlap will provide spontaneous decay channels ${ }^{38,39}$ leading to significant damping of these quasi-particles and therefore to the absence of visible signatures in INS data.

A second important question arises from the likely detection of triplet excitations in IR spectroscopy presented in Sec. II C. Indeed, dominant electric dipole transitions induced by light are strictly confined to spin-conserving excitations $(\Delta S=0)$ and are therefore, in principle, unable to reveal singlet-to-triplet transitions. However, it has been shown that, in a number of low-dimensional quantum magnets, this selection rule can be circumvented through essentially two mechanisms relying on the presence of spin-phonon coupling and involving one or multiple magnetic excitations.

A successful and now well-established model employed to describe the infrared optical absorption of one and two-dimensional undoped cuprates is based on phononassisted bi-magnon absorption ${ }^{34,40-43}$. The excitation of singlet bound states, resulting from the coupling of two spin-carrying modes (triplets, in our case) in such a way that the total spin amounts to zero, indeed obeys the imposed spin selection rule. Lorenzana and Sawatzky further showed that, when a center of inversion is present, dipole-allowed absorption is only possible if a symmetrybreaking phonon is also involved in the process ${ }^{40,41}$. In our case, an attribution of the IR band observed at $14.3 \mathrm{meV}$ for $T<70 \mathrm{~K}$ to the absorption of phononassisted bi-magnons is very unlikely as the typical energy of these excitations, already of the order of $\sim 2 \Delta=$ $21.2 \mathrm{meV}$ when neglecting the phonon energy, are much 

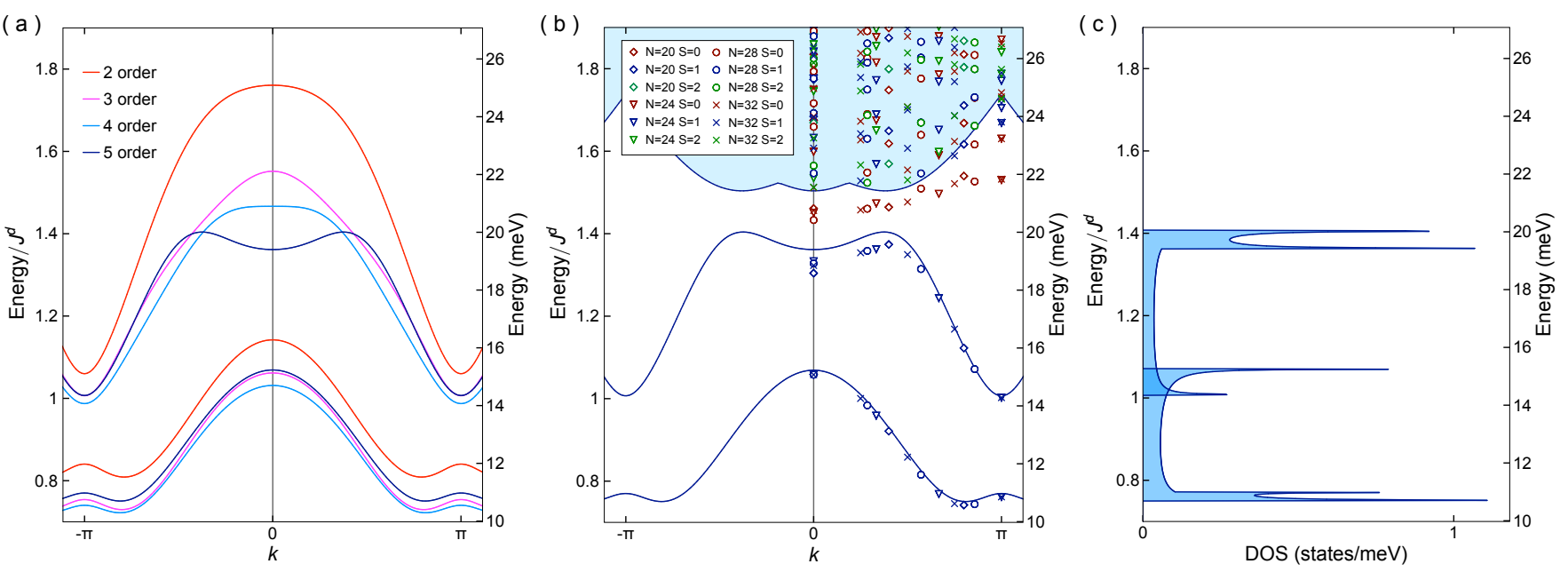

FIG. 7. (a) One-triplet dispersion relation calculated from second to fifth order perturbation theory. (b) Comparison of the one-triplet dispersion relation obtained for the fifth order perturbation theory with exact diagonalization results obtained on finite lattices up to $N=32$ sites. First few lowest-lying singlets (in red) and triplet (in blue) obtained from ED are shown. The blue filled area corresponds to the free two-triplet continuum. (c) One-triplet density of states obtained from fifth order perturbation theory dispersion relations shown in (b). Energies given in meV have been obtained by downscaling the DFT isotropic magnetic couplings by $50 \%$.

larger.

An alternative mechanism, arising from the spin-orbit coupling, has been proposed to explain the detection of singlet-to-triplet excitations in dimerized quantum magnets using IR absorption ${ }^{25,44}$. It can be described qualitatively as a process where light excites the system into a virtual spin-singlet one-phonon state coupled, through a dynamic Dzyaloshinskii-Moriya (DM) interaction, to a spin-triplet zero-phonon state ${ }^{45}$. This mechanism thus relies on the assumption that the virtual polar phonon involved in the process is associated with atomic displacements able to induce an instantaneous variation of the $\mathrm{DM}$ vector. Assuming that such a mechanism is effective in the low-temperature phase of $\mathrm{Li}_{2} \mathrm{Cu}_{2} \mathrm{O}\left(\mathrm{SO}_{4}\right)_{2}$, the $14.3 \mathrm{meV}$ IR absorption band would, quite accurately, match the zone center maximum of the lowest one-triplet excitation and the corresponding Van Hove singularity in the DOS.

\section{CONCLUSION}

In conclusion, we report the first experimental investigation of magnetic excitations in the low-temperature, dimerized phase of the recently discovered frustrated spin- $1 / 2$ two-leg ladder $\mathrm{Li}_{2} \mathrm{Cu}_{2} \mathrm{O}\left(\mathrm{SO}_{4}\right)_{2}$. Through a combined analysis of inelastic neutron scattering, magnetic susceptibility and infrared absorption spectroscopy data obtained on powder samples, dispersive excitations of bandwidth of the order of $5 \mathrm{meV}$ have been clearly identified above a large spin gap of $10.6 \mathrm{meV}$. Exact diagonalization and higher-order perturbation theory calculations allowed for an overall consistent interpretation of these results in terms of one-triplet quasi-particle excita- tions above the singlet ground state. While experiments and theory show an overall good agreement, the only exception lies in the high-energy part of the triplet excitation spectrum, where a possible coupling between the quasi-particles and the high-lying many-particle continuum may be responsible for the absence of high-energy structure in the INS spectra. This calls for further experimental and theoretical investigations of this very rare example of frustrated spin-1/2 ladder, which will heavily rely on the future availability of single crystals.

\section{ACKNOWLEDGMENTS}

This work was supported by French state funds managed by the ANR within the Investissements d'Avenir programme under reference ANR-11-IDEX-0004-02, and more specifically within the framework of the Cluster of Excellence MATISSE led by Sorbonne Universités. The authors thank Dr Meiling Sun for providing the sample used in this study. Inelastic neutron scattering experiments were performed at the Institut Laue-Langevin (ILL) in Grenoble.

\section{Appendix A: Series expansions}

The evaluation of the one-triplet dispersion relation has been carried out solving the spin Hamiltonian parametrized from first-principles around the limit of isolated dimer (low-temperature phase), implementing a high-order perturbative approach in the strong coupling expansion in the spirit of textbooks such as chapter 11 of Ref. 46. 
Following Ref. 18, the triclinic phase can be described by the staggered $S=1 / 2$ dimer structure schematized in Fig. 1(e). In this structure one of the couplings along the legs, $J^{d}$, is much stronger than all the others. The triclinic Hamiltonian $H$ can thus be written as the sum of an unperturbed part $H_{0}$, for decoupled dimers along the legs (bold blue lines in Fig. 1(e)) and a perturbation,
$W$, accounting for the coupling between the dimers with

$$
H_{0}=\sum_{m}\left[J^{d}\left(\mathbf{S}_{1, a}^{m} \cdot \mathbf{S}_{2, a}^{m}+\mathbf{S}_{1, b}^{m} \cdot \mathbf{S}_{2, b}^{m}\right)\right]
$$

and

$$
\begin{aligned}
W= & \sum_{m}\left[J_{\perp}^{a}\left(\mathbf{S}_{1, a}^{m} \cdot \mathbf{S}_{2, b}^{m}\right)+J_{\perp}^{b}\left(\mathbf{S}_{2, a}^{m-1} \cdot \mathbf{S}_{1, b}^{m}\right)+J_{2}\left(\mathbf{S}_{1, a}^{m} \cdot \mathbf{S}_{1, a}^{m+1}+\mathbf{S}_{2, a}^{m} \cdot \mathbf{S}_{2, a}^{m+1}+\mathbf{S}_{1, b}^{m} \cdot \mathbf{S}_{1, b}^{m+1}+\mathbf{S}_{2, b}^{m} \cdot \mathbf{S}_{2, b}^{m+1}\right)\right. \\
& \left.+J^{a}\left(\mathbf{S}_{1, a}^{m} \cdot \mathbf{S}_{2, a}^{m-1}+\mathbf{S}_{1, b}^{m} \cdot \mathbf{S}_{2, b}^{m-1}\right)+J_{\times}^{b}\left(\mathbf{S}_{1, a}^{m-1} \cdot \mathbf{S}_{1, b}^{m}+\mathbf{S}_{2, a}^{m-1} \cdot \mathbf{S}_{2, b}^{m}\right)+J_{\times}^{c}\left(\mathbf{S}_{1, a}^{m} \cdot \mathbf{S}_{1, b}^{m}+\mathbf{S}_{2, a}^{m} \cdot \mathbf{S}_{2, b}^{m}\right)\right],
\end{aligned}
$$

where $m$ is the cell index, $a$ and $b$ denote the two legs of the ladder, the number 1 or 2 distinguishes the upper and the lower spin-site of a dimer and $\mathbf{S}_{i, \alpha}^{m}$ with $\alpha=\{a, b\}$ and $i=\{1,2\}$, are the spin- $1 / 2$ operators.

At $W=0$, the system consists of isolated dimers and the unperturbed ground state corresponds to a product of singlets on the leg dimers. The first excited state is the one-triplet state $|t\rangle_{\alpha}^{m}$, a state with a single triplet on a dimer $(m, \alpha)$ and singlets on all the other dimers. As $\mathrm{Li}_{2} \mathrm{Cu}_{2} \mathrm{O}\left(\mathrm{SO}_{4}\right)_{2}$ contains two dimers per unit cell, a $2 \times 2$ effective Hamiltonian, $W$ has to be computed for each value of $k$ in Fourier space. This leads to two separate bands of triplets. The dispersion relation is obtained by diagonalizing the effective Hamiltonian up to a given order starting from the Bloch states $|T\rangle_{\alpha}=\frac{2}{\sqrt{N_{c}}} \sum_{m} e^{i k m}|t\rangle_{\alpha}^{m}$, where $\alpha=\{a, b\}$ and $N_{c}$ is the number of unit cells.

\section{First order}

At first order, the effective Hamiltonian $\mathbf{W}_{\mathrm{o} 1}$ is given simply by the matrix elements of Eq. (A2) between the states $|T\rangle_{\alpha}$. The diagonal elements $\mathbf{W}_{\mathrm{o} 1}(1,1)=$ $\mathbf{W}_{\mathrm{o} 1}(2,2)$ and the off-diagonal $\mathbf{W}_{\mathrm{o} 1}(2,1)=\mathbf{W}_{\mathrm{o} 1}^{*}(1,2)$ can be found by elementary means and read:

$\mathbf{W}_{\mathrm{o} 1}(1,1)=\left(J_{2}-\frac{J_{a}}{2}\right) \cos k$,
$\mathbf{W}_{\mathrm{o} 1}(1,2)=\frac{1}{4}\left((\cos k-i \sin k)\left(2 J_{\times}^{b}-J_{\perp}^{a}\right)+2 J_{\times}^{c}-J_{\perp}^{b}\right)$.

\section{Second order}

Computation of the second order is already more cumbersome, but can still be performed by elementary means (following for example the strategy of sections IV and V of Ref. 47). The second-order corrections $\mathbf{W}_{\mathrm{o} 2}(1,1)=$
$\mathbf{W}_{\mathrm{o} 2}(2,2)$ and $\mathbf{W}_{\mathrm{o} 2}(2,1)=\mathbf{W}_{\mathrm{o} 2}^{*}(1,2) \operatorname{read}$

$$
\begin{aligned}
\mathbf{W}_{\mathrm{o} 2} & (1,1)=\frac{1}{32}\left(12\left(J_{\times}^{c}-J_{\perp}^{b}\right) J_{\times}^{c}-\left(J_{\perp}^{a}\right)^{2}-\left(J_{\perp}^{b}\right)^{2}\right. \\
& +12\left(J_{\times}^{b}-J_{\perp}^{a}\right) J_{\times}^{b}-2\left(J^{a}\right)^{2}+24\left(J_{2}-J^{a}\right) J_{2} \\
& -2\left(2 J_{2}-J^{a}\right)^{2} \cos (2 k)+2\left(\left(2 J_{\times}^{c}-J_{\perp}^{b}\right) J_{\perp}^{a}\right. \\
& \left.\left.-4\left(J^{a}\right)^{2}-2\left(2 J_{\times}^{c}-J_{\perp}^{b}\right) J_{\times}^{b}\right) \cos k\right)
\end{aligned}
$$



FIG. 8. Exact diagonalization results obtained on finite lattices up to $N=32$ sites. The triplet $(S=1)$ energies for $k=0$ and $k=\pi$ are plotted as a function of $1 / N$. 


$$
\begin{aligned}
& \mathbf{W}_{\mathrm{o} 2}(1,2)=\frac{1}{16}\left(2\left(-\left(J_{\perp}^{b}\right)^{2}+i \sin k\left(J_{\perp}^{a}\right)^{2}\right)-\left(2 J_{2}\right.\right. \\
& \left.\quad-J^{a}\right)\left(2 J_{\times}^{b}-J_{\perp}^{a}\right)-(\cos (2 k)-i \sin (2 k))\left(2 J_{2}\right. \\
& \left.\quad-J^{a}\right)\left(2 J_{\times}^{b}-J_{\perp}^{a}\right)-2\left(\left(2 J_{2}-J^{a}\right)\left(2 J_{\times}^{c}-J_{\perp}^{b}\right)\right. \\
& \left.\left.\quad+\left(J_{\perp}^{a}\right)^{2}\right) \cos k\right) .
\end{aligned}
$$

\section{Fifth order}

To proceed beyond second order, we have adapted a C-code that was used previously, e.g., in Refs. 48 and 49 to the present situation. The main challenges here include the large number of variables (6 coupling ratios) as well as the rather long-range nature of some couplings (see Fig. 1(e)). This limits us to fifth order, the resulting expressions are too cumbersome to be presented here (the full expression of the perturbation matrix $W$ can be found in the Mathematica notebook provided in the Supplemental Material ${ }^{50}$ that also contains a seventhorder series for the ground-state energy). Nevertheless, we note that there is a freedom of the choice of basis and implementation of the perturbation theory that starts to impact the effective Hamiltonian $W$ at order three. For efficiency reasons, we decided to implement the perturbative corrections to the basis vectors starting from $|T\rangle_{\alpha}$ such that orthonormality is lost at higher order. As a consequence, the matrix representation of $W$ no longer needs to be Hermitian and, indeed, we find complex diagonal matrix elements starting at order 3 . Nevertheless, we have checked that the eigenvalues of this $2 \times 2$ matrix are always real, as they should be since they are independent of the choice of basis.

We have also double-checked that we recover known limiting cases from our 6-variable expressions: for $J_{\perp}^{a}=$ $J_{\perp}^{b}=J^{a}=J_{2}=0$ and $J_{\times}^{b}=J_{\times}^{c}$ we recover the conventional two-leg ladder, and our results for the eigenvalues are indeed consistent with Ref. 51; for $J_{\perp}^{a}=J_{\perp}^{b}=J_{\times}^{b}=$ $J_{\times}^{c}=0$ our model specializes to two decoupled frustrated and dimerized chains, a system that has been studied in Ref. 52 and Ref. 53 for $J_{2}=0$.
For illustration purposes, we report a numerical expression of the dispersion relation up to full fifth order, obtained by substituting the DFT values of the coupling constants given in section III

$$
\begin{aligned}
& \omega_{\mathrm{o} 5 \pm}(k)=1.09+1.65 \cdot 10^{-1} \cos (k)-3.54 \cdot 10^{-2} \cos (2 k) \\
& \quad-1.60 \cdot 10^{-3} \cos (3 k)-2.68 \cdot 10^{-3} \cos (4 k) \\
& \quad+1.54 \cdot 10^{-4} \cos (5 k) \pm\left(4.07 \cdot 10^{-2}-8.75 \cdot 10^{-4} \cos (k)\right. \\
& \quad-2.35 \cdot 10^{-2} \cos (2 k)+4.38 \cdot 10^{-3} \cos (3 k) \\
& \quad+7.68 \cdot 10^{-4} \cos (4 k)+1.94 \cdot 10^{-4} \cos (5 k) \\
& \quad-1.20 \cdot 10^{-4} \cos (6 k)-2.27 \cdot 10^{-6} \cos (7 k) \\
& \left.\quad+1.04 \cdot 10^{-6} \cos (8 k)+7.15 \cdot 10^{-7} \cos (9 k)\right)^{1 / 2} .
\end{aligned}
$$

For reasons of compactness of presentation, we have rounded the numerical coefficients to three significant digits. Expression (A7) has been used in Fig. 7 for order five in panel (a) and systematically in panel (b).

\section{Appendix B: Finite-size effects}

Here we take a closer look at the finite-size effects present in the numerical results for the one-triplet dispersion shown in section III. Indeed, Fig. 7(b) exhibits a remarkable convergence of the numerical results towards the fifth-order perturbative expansion with increasing $N$. Figure 8 shows the ED energies of the triplet excitations for two different values of $k, k=0$ and $k=\pi$, as a function of the inverse of the size, $1 / N$, including the smaller system sizes $N=12$ and 16 not shown previously. One observes that the values of the energies for $k=\pi$ and for the lower band of the triplet at $k=0$ converge rapidly. Larger finite-size effects are only observed at the top of the upper band at $k=\pi$. This corresponds to the region where the series also show a slow convergence (see Fig. 7(a)) and we speculate that this is again due to the proximity with the continuum. Still, for systems with $N>20$, the data can also be considered to converge to the thermodynamic limit. Even in this least favorable case, finite-size corrections to the $N=32$ data are presumably negligible for both bands and all values of $k$.
* guillaume.radtke@sorbonne-universite.fr

${ }^{1}$ C. Lacroix, P. Mendels, and F. Mila, Introduction to Frustrated Magnetism: Materials, Experiments, Theory, Springer Series in Solid-State Sciences, Vol. 164 (Springer Berlin Heidelberg, 2011).

${ }^{2}$ F. Mila, Eur. Phys. J. B 6, 201-205 (1998).

3 A. Honecker, F. Mila, and M. Troyer, Eur. Phys. J. B 15, 227-233 (2000).

4 T. Vekua and A. Honecker, Phys. Rev. B 73, 214427 (2006).
${ }^{5}$ G.-H. Liu, H.-L. Wang, and G.-S. Tian, Phys. Rev. B 77, 214418 (2008).

6 R. Wen, G.-H. Liu, and G.-S. Tian, Commun. Theor. Phys. 55, 1102-1108 (2011).

7 Y.-C. Li and H.-Q. Lin, New J. Phys. 14, 063019 (2012).

8 A. A. Tsirlin, I. Rousochatzakis, D. Kasinathan, O. Janson, R. Nath, F. Weickert, C. Geibel, A. M. Läuchli, and H. Rosner, Phys. Rev. B 82, 144426 (2010).

9 B. Koteswararao, S. Salunke, A. V. Mahajan, I. Dasgupta, and J. Bobroff, Phys. Rev. B 76, 052402 (2007). 
10 O. Mentré, E. Janod, P. Rabu, M. Hennion, F. LeclercqHugeux, J. Kang, C. Lee, M.-H. Whangbo, and S. Petit, Phys. Rev. B 80, 180413 (2009).

${ }^{11}$ K. W. Plumb, Z. Yamani, M. Matsuda, G. J. Shu, B. Koteswararao, F. C. Chou, and Y.-J. Kim, Phys. Rev. B 88, 024402 (2013).

12 K. W. Plumb, K. Hwang, Y. Qiu, L. W. Harriger, G. E. Granroth, A. I. Kolesnikov, G. J. Shu, F. C. Chou, C. Rüegg, Y. B. Kim, and Y.-J. Kim, Nat. Phys. 12, 224-229 (2016).

13 Y. Kohama, S. Wang, A. Uchida, K. Prsa, S. Zvyagin, Y. Skourski, R. D. McDonald, L. Balicas, H. M. Ronnow, C. Rüegg, and M. Jaime, Phys. Rev. Lett. 109, 167204 (2012).

14 F. Casola, T. Shiroka, A. Feiguin, S. Wang, M. S. Grbić, M. Horvatić, S. Krämer, S. Mukhopadhyay, K. Conder, C. Berthier, H.-R. Ott, H. M. Rønnow, Ch. Rüegg, and J. Mesot, Phys. Rev. Lett. 110, 187201 (2013).

15 Y. Kohama, K. Mochidzuki, T. Terashima, A. Miyata, A. DeMuer, T. Klein, C. Marcenat, Z. L. Dun, H. Zhou, G. Li, L. Balicas, N. Abe, Y. H. Matsuda, S. Takeyama, A. Matsuo, and K. Kindo, Phys. Rev. B 90, 060408 (2014).

16 M. Sun, G. Rousse, A. M. Abakumov, M. Saubanère, M.L. Doublet, J. Rodríguez-Carvajal, G. Van Tendeloo, and J.-M. Tarascon, Chem. Mater. 27, 3077-3087 (2015).

17 G. Rousse, J. Rodríguez-Carvajal, C. Giacobbe, M. Sun, O. Vaccarelli, and G. Radtke, Phys. Rev. B 95, 144103 (2017).

18 O. Vaccarelli, G. Rousse, A. Saúl, and G. Radtke, Phys. Rev. B 96, 180406 (2017).

19 D. Richard, M. Ferrand, and G.J. Kearley, J. Neutron Research 4, 33-39 (1996).

20 S. W. Lovesey, Theory of Neutron Scattering from Condensed Matter (Clarendon Press, Oxford UK, 1984).

21 G. L. Squires, Introduction to the Theory of Thermal Neutron Scattering (Dover Publications, 1978).

22 J. P. Clancy, B. D. Gaulin, C. P. Adams, G. E. Granroth, A. I. Kolesnikov, T. E. Sherline, and F. C. Chou, Phys. Rev. Lett. 106, 117401 (2011).

23 M. Troyer, H. Tsunetsugu, and D. Würtz, Phys. Rev. B 50, 13515-13527 (1994).

24 V. C. Farmer, The Infrared Spectra of Minerals (Mineralogical Society of Great Britain and Ireland, 1974).

25 T. Rõõm, D. Hüvonen, U. Nagel, J. Hwang, T. Timusk, and H. Kageyama, Phys. Rev. B 70, 144417 (2004).

26 A. Furrer and H. U. Güdel, J. Magn. Mag. Mat. 14, 256 (1979).

27 P. C. Hohenberg and W. F. Brinkmann, Phys. Rev. B 10, 128 (1974).

28 M. B. Stone, Y. Chen, J. Rittner, H. Yardimci, D. H. Reich, C. Broholm, D. V. Ferraris, and T. Lectka, Phys. Rev. B 65, 064423 (2002).

29 D. L. Quintero-Castro, B. Lake, E. M. Wheller, A. T. M. N. Islam, T. Guidi, K. C. Rule, Z. Izaola, M. Russina,
K. Kiefer, and Y. Skourski, Phys. Rev. B 81, 014415 (2010).

30 E. Canévet, B. Fåk, R. K. Kremer, J. H. Chun, M. Enderle, E. E. Gordon, J. L. Bettis, M.-H. Whangbo, J. W. Taylor, and D. T. Adroja, Phys. Rev. B 91, 060402(R) (2015).

31 C. Lanczos, J. Res. Nat. Bur. Stand. 45, 255 (1950).

32 E. Dagotto, Rev. Mod. Phys. 66, 763 (1994).

33 S. Trebst, H. Monien, C. J. Hamer, Z. Weihong, and R. R. P. Singh, Phys. Rev. Lett. 85, 4373-4376 (2000).

${ }^{34}$ M. Windt, M. Grüninger, T. Nunner, C. Knetter, K. P. Schmidt, G. S. Uhrig, T. Kopp, A. Freimuth, U. Ammerahl, B. Büchner, and A. Revcolevschi, Phys. Rev. Lett. 87, 127002 (2001).

35 R. L. Martin and F. Illas, Phys. Rev. Lett. 79, 1539-1542 (1997).

36 A. Saúl and G. Radtke, Phys. Rev. B 89, 104414 (2014).

37 H. Jeschke, I. Opahle, H. Kandpal, R. Valentí, H. Das, T. Saha-Dasgupta, O. Janson, H. Rosner, A. Brühl, B. Wolf, M. Lang, J. Richter, S. Hu, X. Wang, R. Peters, T. Pruschke, and A. Honecker, Phys. Rev. Lett. 106, 217201 (2011).

38 M. E. Zhitomirsky, Phys. Rev. B 73, 100404 (2006).

39 M. E. Zhitomirsky and A. L. Chernyshev, Rev. Mod. Phys. 85, 219-242 (2013).

40 J. Lorenzana and G. A. Sawatzky, Phys. Rev. Lett. 74, 1867-1870 (1995).

41 J. Lorenzana and G. A. Sawatzky, Phys. Rev. B 52, 9576 9589 (1995).

42 J. Lorenzana and R. Eder, Phys. Rev. B 55, R3358-R3361 (1997).

43 H. Suzuura, H. Yasuhara, A. Furusaki, N. Nagaosa, and Y. Tokura, Phys. Rev. Lett. 76, 2579-2582 (1996).

44 T. Rõõm, D. Hüvonen, U. Nagel, Y.-J. Wang, and R. K. Kremer, Phys. Rev. B 69, 144410 (2004).

45 O. Cépas and T. Ziman, Phys. Rev. B 70, 024404 (2004).

46 G. Baym, Lectures on Quantum Mechanics (Westview Press, 1969).

47 J. B. Kogut, Rev. Mod. Phys. 51, 659-713 (1979).

48 D. C. Cabra, A. Honecker, and P. Pujol, Phys. Rev. B 58, 6241-6257 (1998).

49 A. Honecker, Phys. Rev. B 59, 6790-6794 (1999).

50 See ancillary files for the expression of the effective Hamiltonian up to the fifth order and the ground state energy up to the seventh order in the form of a Mathematica notebook, PDF, or plain text.

51 M. Reigrotzki, H. Tsunetsugu, and T. M. Rice, J. Phys.: Condens. Matter 6, 9235-9245 (1994).

52 C. Knetter and G. S. Uhrig, Eur. Phys. J. B 13, 209-225 (2000).

53 T. Barnes, J. Riera, and D. A. Tennant, Phys. Rev. B 59, 11384-11397 (1999). 